\title{
Venetoclax in the Treatment of Chronic Lymphocytic Leukemia: Evidence, Expectations, and Future Prospects
}

\author{
Saba Tariq ${ }^{1}$, Sundus Tariq ${ }^{2}$, Maliha Khan ${ }^{3}$, Aysha Azhar ${ }^{4}$, Mukhtiar Baig ${ }^{5}$
}

1. Pharmacology and Therapeutics, The University of Faisalabad, Faisalabad, PAK 2. Physiology, The University of Faisalabad, Faisalabad, PAK 3. Oncology, The University of Texas MD Anderson Cancer Center, Houston, USA 4. Post Graduate Research Department, Madina Teaching University, Faisalabad, PAK 5. Clinical Biochemistry, King Abdulaziz University, Jeddah, SAU

Corresponding author: Saba Tariq, drsabatariq1@gmail.com

\begin{abstract}
Chronic lymphocytic leukemia (CLL) is the most common form of leukemia in the western adult population; it is also prevalent worldwide. The B cell lymphoma-2 (BCL-2) family proteins play a key role in regulating intrinsic apoptosis and, in many cancers, are the main culprits behind tumor survival and therapy resistance. Hence, the role of BCL-2 inhibitors is very beneficial in the treatment of CLL. Venetoclax is the first selective, orally bioavailable BCL-2 inhibitor.

This review article discusses factors such as the pharmacokinetics, pharmacodynamics, acquired resistance to venetoclax, responders vs. non-responders in venetoclax monotherapy, and the synergistic role of venetoclax with other drugs in detail. Venetoclax is the first BH3 mimetic drug and selective BCL-2 inhibitor that has received FDA approval. This drug has proved to provide good therapeutic responses in CLL patients irrespective of the presence of adverse clinical or genetic features, including in patients with relapsed or refractory forms of CLL. We anticipate that novel combination therapies, including venetoclax and immunotherapy, will further alter the treatment landscape for patients with relapsed CLL, particularly for those with deletion $17 \mathrm{p}$ (del 17p) CLL, which carries a very poor prognosis.
\end{abstract}

Categories: Miscellaneous, Oncology

Keywords: venetoclax, chronic lymphocytic leukemia, pharmacokinetics, pharmacodynamics

\section{Introduction And Background Chronic lymphocytic leukemia}

Chronic lymphocytic leukemia (CLL) is a hematological monoclonal neoplastic disorder. It is the most common form of leukemia in the western adult population [1], accounting for approximately $30 \%$ of all leukemias in this group [2]. It is characterized by the proliferation of incompetent, poorly formed, and dysfunctional CD5/CD23-B lymphocytes, thereby leading to their accumulation in the peripheral blood, lymphoid tissues, and bone marrow, resulting in lymphocytosis, lymphadenopathy, splenomegaly, and leukemia cell infiltration of the marrow [3].

Received 06/19/2020 Review ended 06/23/2020 Published 06/29/2020

\section{(c) Copyright 2020}

Tariq et al. This is an open access article distributed under the terms of the Creative Commons Attribution License CC-BY 4.0., which permits unrestricted use, distribution, and reproduction in any medium, provided the original author and source are credited.
CLL represents $22-30 \%$ of all leukemias worldwide, with an incidence between $<1$ and 5.5 per 100,000 people per year. According to a study carried out in 2004, the countries with the highest incidence rates were Australia, the United States (US), Ireland, and Italy [4]. In the US, the annual incidence of CLL is nearly 4.6 cases per 100,000 persons/year, with 4,500 deaths and $>15,000$ newly diagnosed cases reported per year. In 2015, there were approximately 14,620 new CLL cases reported in the US alone [5]. More than 95\% of patients were older than 50 years, with a median age at diagnosis of 71 years [2]. The occurrence is slightly more common in males than in females of the same age group [3] and is less frequent in individuals with Asian and Middle Eastern ancestry [6].

\section{Treatment strategy}

Multiple factors must be taken into consideration before treatment selection. These include the patient's condition at diagnosis, clinical stage of the disease, response to previous chemotherapy, and the molecular and cytogenetic makeup of the patient. For example, in those with deletion 17p (del 17p) CLL, options that lead to therapeutic responses are fewer; hence, selecting a suitable treatment option for this population is critical [7]. A wide variety of treatment options are available for patients with CLL. These include chemotherapy, a combination of chemotherapy and immunotherapy, and drugs that target the signaling pathways that facilitate the growth and survival of CLL cells [e.g., B cell antigen receptor (BCR) signaling and B-cell lymphoma-2 (BCL-2)] [8]. Currently, the first-line treatment for patients in good condition and without significant comorbidities is predominately chemotherapy (chlorambucil, fludarabine, cyclophosphamide, and bendamustine) and combination therapy with monoclonal antibodies to CD20 
(rituximab). According to the CLL 3 Trial, the fludarabine plus cyclophosphamide (FC) and cladribine plus cyclophosphamide (CC) regimens have similar therapeutic efficacy as the first-line treatment of CLL [9].

Proto-oncogenes, particularly BCL-2 genes, are mainly responsible for the resistance to programmed cell death seen in patients with CLL [10]. It is interesting to note that BCL-2 expression is increased in $95 \%$ of the patients with CLL [11]. One of the important factors that result in overexpression of BCL-2 is the hypomethylation of the BCL-2 gene, which leads to an increase in the activity, as indicated by histone H3 lysine 27 (H3K27) acetylation chromatin analysis. The absence of micro RNA due to post-transcriptional regulation and an increase in the expression of myeloid cell leukemia 1 (MCL1) protein maybe the other mechanisms of resistance $[12,13]$. BCL-2 overexpression results in the formation of aberrant signaling pathways, which lead to the proliferation and survival of BCL-2 cells. This has been explained by a transgenic mouse model of $\mathrm{t}(14 ; 18)$ translocation where the overexpression of BCL-2 led to the activation of the nuclear factor-kB (NFkB) pathway or the overexpression of tumor necrosis factor (TNF)-receptorassociated factor2 (TRAF2) led to the activation of NF-kB and c-Jun N-terminal kinase (JNK). It was found that only those mice with both $\mathrm{t}(14 ; 18)$ and TRAF2 overexpression developed an aggressive form of CLL [14]. This model closely mimics CLL, in which NF-kB is also inherently activated [15].

\section{Venetoclax}

BCL-2 family proteins play a crucial role in regulating intrinsic apoptosis and are the main culprits behind tumor survival and therapy resistance in many cancers $[16,17]$. Therefore, the role of BCL-2 inhibitors is very beneficial in the treatment of CLL [18-21]. Venetoclax is the first selective, orally bioavailable BCL-2 inhibitor. The molecular weight for venetoclax is $868 \mathrm{Da}$. The main advantage of venetoclax over other agents is that it has a high binding affinity for BCL-2 receptors and it very selectively inhibits BCL-2, maintaining anti-apoptotic activity in cancer cells (Figure 1)[22]. Monotherapy with this agent facilitates a rapid reduction in the disease burden with a high overall response of about $80 \%$ and a complete response of 6-20\% in patients with relapsed or refractory CCL, including those with chromosome $17 \mathrm{p}$ deletions $[18,20]$.

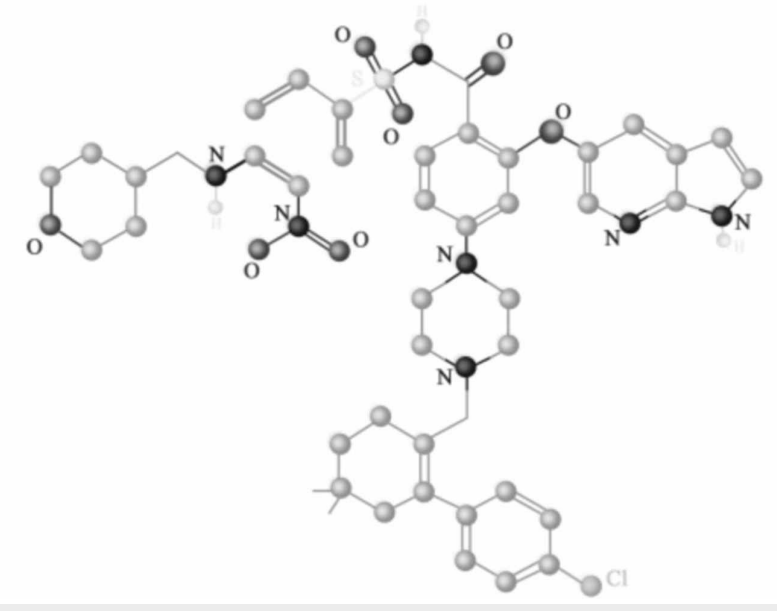

FIGURE 1: Structure of venetoclax

\section{Review}

\section{Pharmacokinetics}

The study of chemotherapeutic drugs on healthy volunteers has always been a daunting task. However, phase I and II trials have provided useful insights into the pharmacology of venetoclax. The half-life of venetoclax after administration of a single dose of $50 \mathrm{mg}$ is about 16-19 hours, and it is highly plasma protein-bound (>99\%) $[18,23,24]$. Clinical trials have shown that a steady-state is typically achieved within a week with daily dosing, associated with minor, non-problematic accumulation in the body [23,24]. In a study conducted on 505 subjects, the volumes of distributions at steady-state were calculated at 321 (95\% CI: 288340 ) and 256 (95\% CI: 228-276) in the male and female cancer patients, respectively. This indicates its large volume of distribution and extensive tissue binding capacity; and It is predominately metabolized by CYP3A. Apparently, total clearance of the drug from plasma after oral administration $(\mathrm{Cl} / \mathrm{F})$ decreases to $19 \%$ in the case of moderate CYP3A inhibitors, and it can further decrease to $84 \%$ in the case of potent CYP3A inhibitors [25].

The bioavailability of venetoclax is dependent on food and dosage [26]. Peak concentrations are achieved after four to five hours of administration in fasting patients and delayed by approximately two hours when taken with a meal. The maximum concentration (Cmax) and area under the curve (AUC) are also raised by three to five folds when taken with food, especially a high-fat meal [27]. The probable explanation for this 
effect is that the lipid content in food increases the intestinal lymphatic transport of the drug [28]. This increases the fraction of drug absorbed and bypasses the hepatic first-pass effect, thus increasing the amount of drug reaching the systemic circulation. Therefore, it is recommended that venetoclax be administered once daily with a meal, preferably one rich in fat, as it increases the bioavailability by 4.25 fold [27]. Acidreducing agents such as omeprazole and cimetidine do not decrease the bioavailability of venetoclax, and factors such as body weight, age, and race affect its pharmacokinetics $[24,25]$. No dose adjustment is required in mild to moderate hepatic or renal impairment [29]; however, the clearance in severe abnormalities of kidney or liver function has not yet been studied. There is minimal urinary excretion of intact venetoclax [23].

\section{Dosage}

Dosage of the drug is administered according to a weekly ramp-up schedule over five weeks to the recommended daily dose of $400 \mathrm{mg}$. The five-week ramp-up dosing schedule is designed to reduce tumor burden. In this ramp-up schedule, the dose is started at $20 \mathrm{mg}$ in week one and is increased to 50, 100, 200, and $400 \mathrm{mg}$ in weeks two, three, four, and five, respectively [30].

\section{Pharmacodynamics}

Programmed cell death is regulated by the BCL-2 family of proteins. In healthy B-lymphocytes, unnecessary apoptosis is prevented by keeping the death mediators, BAX and BAK, in strict check by BCL-2 and other pro-survival/anti-apoptotic proteins [BCL-2L1 (BCL-XL) or MCL1] (Figure 2) [31-33].

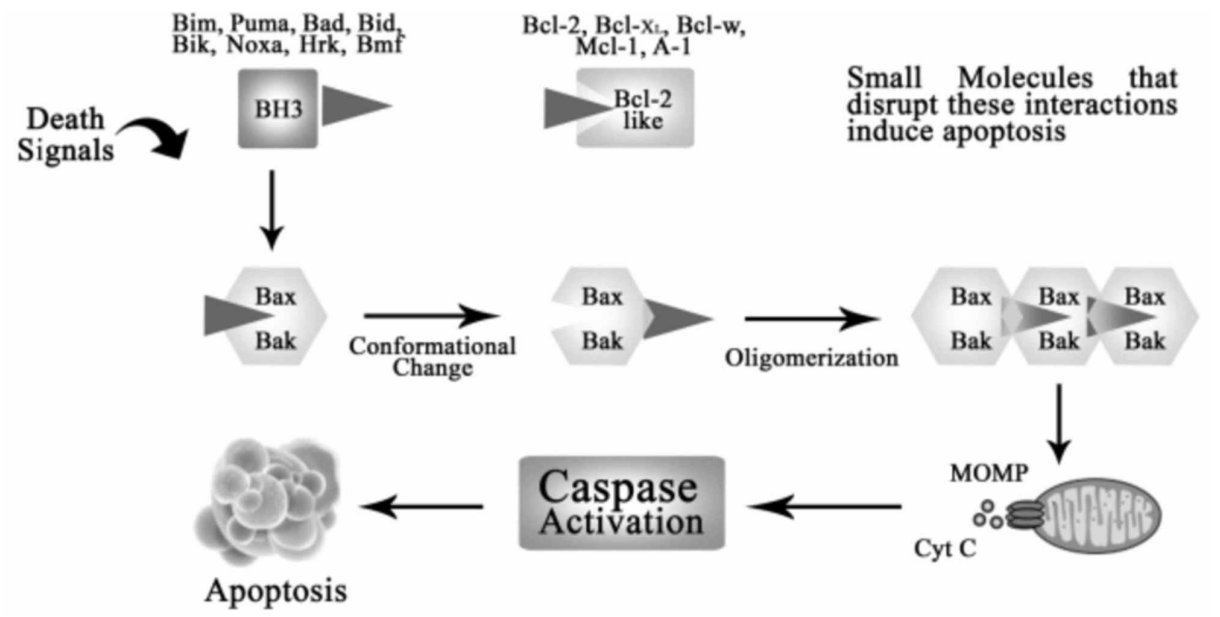

FIGURE 2: Regulation of apoptosis by BCL-2 family

MOMP: mitochondrial outer membrane permeabilization; BCL-2: B-cell lymphoma-2; Cyt C: cytochrome c

However, when the cells are no longer required, they undergo significant irreversible damage, and apoptosis is initiated by activation of BH3- only proteins (BIM, PUMA), which are naturally occurring antagonists of pro-survival BCL-2 proteins. These pro-apoptotic proteins bind and inactivate BCL-2 and its related prosurvival proteins [34], rendering $\mathrm{BAX}$ and $\mathrm{BAK}$ free to cause mitochondrial damage and activate intrinsic pathways of apoptosis. In the case of CLL, the overexpression BCL-2 leads to inappropriate cell survival [35], tumor formation [36], and diminished sensitivity to chemotherapy [37]. Since the antagonistic role of BH3-only proteins towards the BCL-2 is a pivotal step for initiating apoptosis, agents that potently mimic this action were developed to pharmacologically inhibit the pro-survival proteins and initiate programmed cell death in CLL [38].

Initially, a drug named navitoclax was designed. This drug could inhibit BCL-2 receptors as well as B-cell lymphoma-extra large (BCL-XL). BCL-XL also possesses anti-apoptotic activity and is encoded by the BCL-2 genes. In early clinical trials, the dose of navitoclax had to be limited because of the potential side effects of thrombocytopenia. Later, it was discovered that the thrombocytopenia was secondary to the navitoclaxmediated inhibition BCL-XL and was independent of the dose. This necessitated the need for a more specific agent that could selectively inhibit BCL-2 without affecting BCL-XL. Later on, venetoclax was designed to mediate apoptosis in cells with overexpression of BCL-2 without the effects of thrombocytopenia [39]. A significant number of CLL patients show increased responsiveness when treated with chemotherapy, the mechanism of action for which is the induction of apoptosis through tumor protein p53 (TP53). Clonal evolution can lead to mutations in as much as half of the patients in a relapsed or refractory disease setting.

A mutation in TP53 or deletion of parts of chromosome 17 can impair the TP53 function. As a result, the cells' ability to sense DNA damage caused by cytotoxic agents, and the initiation of an appropriate apoptotic 
response greatly decreases [40]. In an in vitro study, venetoclax was found to kill CLL cells, murine lymph node B cells, and RS4;11 human lymphoblast cell lines irrespective of TP53 deletion, mutation, or function. The study further demonstrated that TP53 status does not affect clinical parameters of response to venetoclax [41]. Therefore, as per in vitro and in vivo studies, venetoclax acts independently of TP53 to rapidly induce apoptosis of CLL.

\section{Acquired resistance to venetoclax}

Resistance is one of the major problems in cancer chemotherapy. There are many possible hypotheses for the resistance of venetoclax. One of the most likely mechanisms of resistance is the up-regulation of other proteins members of the BCL-2 anti-apoptotic family. These members include BCL-2A1, BCL-XL, BCL-W, and MCL [42]. Stimulation with CD40 and interleukin-4 (IL-4) causes overexpression of BCL-XL in CLL patients. This can lead to resistance to much higher doses of venetoclax [42]. Another hypothesis has stated that resistance is mainly because of proliferation centers that send kinase-mediated survival signals. Another factor in the resistance to venetoclax is individual variation in these signals. These signals may upregulate anti-apoptotic proteins, as described earlier [43]. One of the important strategies to overcome resistance is to switch between different therapies. Patients with venetoclax resistance show good responses when treated with dasatinib and ibrutinib [42]. Sunitinib is a small molecule, orally available and approved by the FDA for renal cell carcinoma. Literature shows that sunitinib inhibits multiple tyrosine kinase receptors and can be used to overcome venetoclax resistance in CLL patients. It has been found to be more effective than dasatinib and ibrutinib in overcoming resistance to this drug [44]. Nevertheless, it seems the venetoclax resistance might be challenging to overcome with any agent over time. Therefore, it is essential to continue designing and discovering such novel agents that contribute to improvements in chemotherapy.

\section{Responders vs. non-responders in venetoclax monotherapy}

Very few clinical trials have been undertaken with venetoclax alone or in combination with other drugs, and they have shown reasonable response rates. Roberts et al. (2016) performed an open-label, multicenter, phase I/II, monotherapy clinical trial in patients with relapsed or refractory CLL, small lymphocytic lymphoma (SLL), or non- Hodgkin's lymphoma. The study was divided into two phases. In the doseescalation phase, 56 patients received active treatment in one of eight dose groups that ranged from 150 to $1,200 \mathrm{mg}$ of venetoclax per day, out of which three developed tumor lysis syndrome (TLS), leading to one death. Sixty additional patients were treated with a weekly stepwise ramp-up dose in an expansion cohort, increasing from $20,50,100$, and $200 \mathrm{mg}$ to the final recommended dose of $400 \mathrm{mg}$ per day. No TLS was seen in any patients in this phase. Other main adverse events were diarrhea (52\%), upper respiratory tract infection (URTI) (56\%), nausea (55\%), neutropenia (52\%), fatigue (46\%), and cough (35\%). The overall response rate (ORR) with venetoclax in all patients in the escalation cohort and expansion cohort was $79 \%$, $77 \%$, and $82 \%$, respectively, while the complete response rate (CRR) was $20 \%, 30 \%$, and $10 \%$, respectively. The ORR was better in patients of $<70$ years of age $(83 \%)$ than with patients of $\geqslant 70$ years in age $(71 \%)$. Patients previously resistant to fludarabine showed an ORR of $79 \%$ and CRR of $16 \%$. Patients who were not resistant to fludarabine showed better ORR and CRR of $82 \%$ and $27 \%$, respectively. Among all the patients who had a response, the estimated durability of response was $75 \%$ (95\% CI: 64-84) at 15 months. The overall survival estimate during the two-year duration for all the patients was $84 \%$ [18].

Another multicenter, monotherapy, phase 2 , single-arm clinical trial with venetoclax in patients with relapsed or refractory del(17p) CLL, was carried out by Stilgenbauer et al. (2016). In this trial, 107 patients were put in the main cohort and 50 in the safety expansion cohort to evaluate the safety and updated tumor lysis prophylaxis and management measures. Patients were administered venetoclax once daily with a weekly ramp-up schedule of four to five weeks (20,50, 100, 200, and $400 \mathrm{mg}$ ) followed by $400 \mathrm{mg}$ per day, which was continuous dosing until disease progression or discontinuation for another reason. The most common grade 3-4 adverse events were neutropenia (40\%), infection (20\%), anemia (18\%), and thrombocytopenia (15\%). Serious adverse events occurred in 59 (55\%) patients, irrespective of their relationship to treatment, with the most common ( $\geqslant 5 \%$ of patients) being pyrexia and autoimmune hemolytic anemia (7\%), pneumonia (6\%), and febrile neutropenia (5\%). Eleven patients died in the study within 30 days of the last dose of venetoclax: seven due to disease progression and four from an adverse event (none assessed as treatment-related). The ORR was $85 \%$, indicating that this monotherapy can be tolerated well and is active in relapsed or refractory CLL patients [20].

Jones et al. (2015) performed a phase II, double arm, venetoclax monotherapy clinical trial in 28 patients with relapsed or refractory CLL. Twenty-two patients previously treated with ibrutinib for a median duration of 15.5 months entered in arm A, and six patients previously treated with idelalisib entered in arm B for a median duration of 9.7 months. Patients were administered venetoclax once daily $(20,50,100,200$, and 400 $\mathrm{mg}$ ) over five weeks with a weekly ramp-up plan. Adverse events seen in more than $25 \%$ of treatment subjects were neutropenia (57\%), anemia (35\%), nausea (32\%), and diarrhea (32\%). The grade $3-4$ adverse events seen in more than $10 \%$ of treatment subjects were neutropenia (43\%), anemia (29\%), and thrombocytopenia (18\%). The ORR was 53\% [45] (Table 1). 


\section{Cureus}

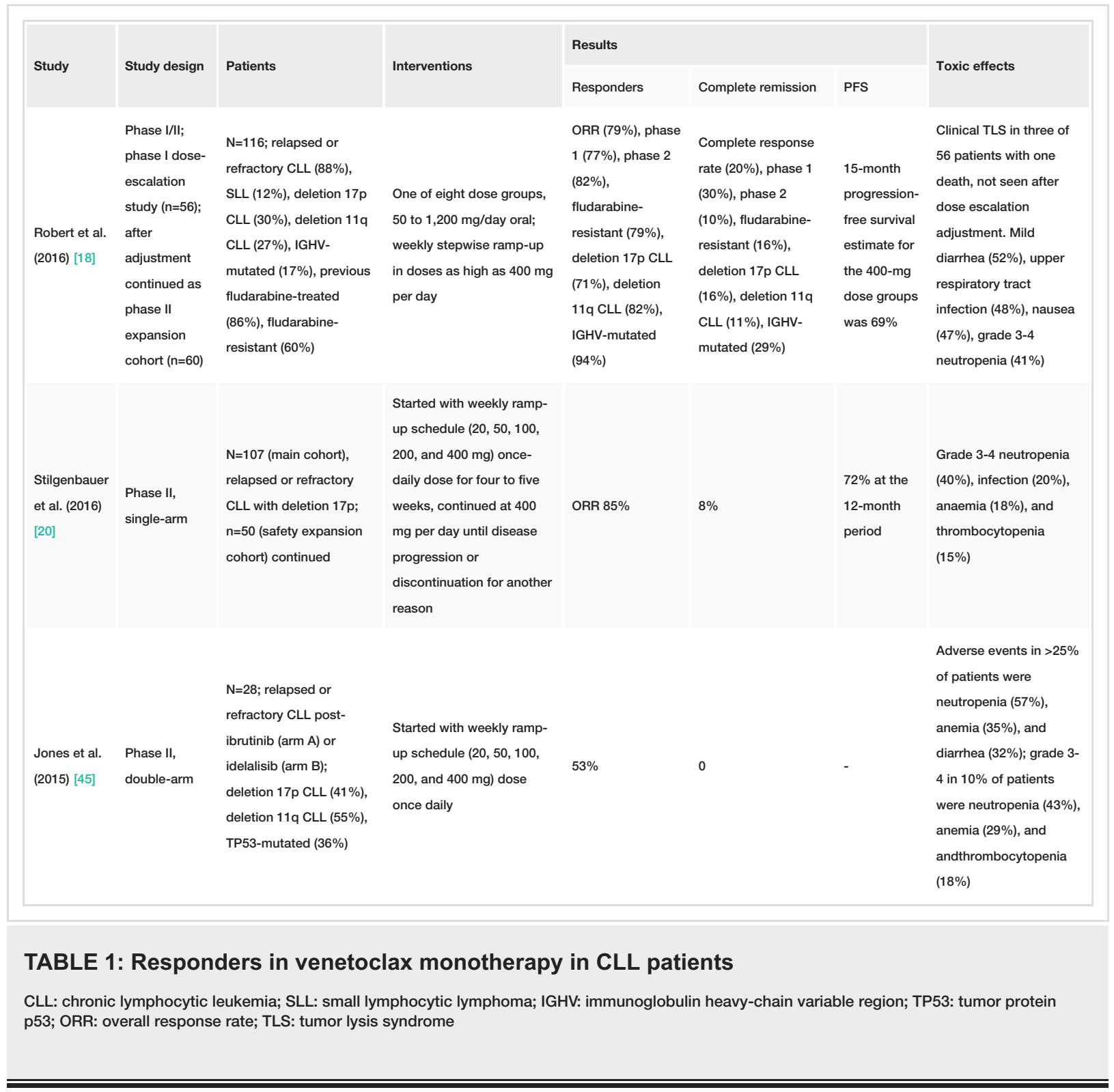

\section{Synergistic role of venetoclax with other drugs}

The treatment protocols of relapsed or refractory CLL are changing due to the development of resistance to monotherapy and also with a view to improving the patient outcome of this disease as it continues to have a relatively poor prognosis despite the wide variety of treatment options available. Venetoclax monotherapy, as shown in the previous trials, is active and well-tolerated; but unfortunately, experimental evidence has shown mutations in the BCL-2, BH3 domain, and C-terminal transmembrane domain of BAX on continuous exposure to venetoclax, thereby leading to ultimate resistance [46]. The synergistic role of venetoclax has also been observed in combination with other drugs in various clinical trials. A phase Ib trial of venetoclax with bendamustine/rituximab (VEN+BR) or bendamustine/obinutuzumab (VEN+BG) was conducted by Stilgenbauer et al. (2016) in patients with relapsed/refractory $(R / R)(n=30)$ or previously untreated CLL (1L) $(n=25)$. The treatment was started with six months of combination therapy in two schedules: A and B. In schedule A, venetoclax was administered before BR or BG and afterward in schedule B. Venetoclax 100 to $400 \mathrm{mg} /$ day in a gradual ramp-up fashion was given with BR/BG in three+three dose-escalation cohort, followed by a safety expansion cohort of $400 \mathrm{mg}$, continued as a single-agent VEN until unacceptable toxicity, disease advancement, or up to one-year total VEN [47]. The adverse events seen in $>20 \%$ of the 47 patients who received VEN+BR (30 R/R; 12 on schedule A at doses $100-400 \mathrm{mg}, 18$ on schedule B at $400 \mathrm{mg}$ ) include neutropenia (63\%), thrombocytopenia (47\%), any infections and infestations (73\%), anemia (43\%), and diarrhea (43\%). Grade 3-4 adverse events were seen in $>10 \%$ of the patients and included neutropenia (63\%), thrombocytopenia (27\%), any infections and infestations (27\%), anemia (20\%), and diarrhea (10\%). The adverse events that were seen in >20\% of the 47 patients who received VEN+BR (17 1L, all at $400 \mathrm{mg}$ : six on schedule A, 11 on schedule B) were neutropenia (76\%), thrombocytopenia (59\%), any infections and infestations (53\%), anemia (35\%), and diarrhea (29\%); grade 3-4 adverse events seen in $>10 \%$ of the patients included neutropenia (71\%), thrombocytopenia (24\%), any infections and infestations (0\%), anemia (29\%), 
The adverse events seen in $>20 \%$ of the eight patients who received VEN+BG (all on schedule B at $400 \mathrm{mg}$ ) were neutropenia (25\%), thrombocytopenia (63\%), any infections and infestations (63\%), anemia (0\%), and diarrhea (38\%). In comparison, grade $3-4$ adverse events seen in $>10 \%$ of the patients were neutropenia (25\%) and thrombocytopenia (63\%). Venetoclax was discontinued early due to toxicity (mainly due to neutropenia and thrombocytopenia) in seven patients on VEN+BR (R/R), two patients on VEN+BR (1L), and one patient on VEN+BG. Combination drugs, bendamustine, rituximab, and/or obinutuzumab were discontinued in 10 patients with VEN+BR (R/R), four patients on VEN+BR (1L), and two patients on VEN+BG. A median of four cycles of $B$ was completed even with early discontinuation. The response was seen in all evaluated patients, with complete remission (CR) in more than half of patients with manageable toxicities and no TLS [47].

Fischer et al. (2016) evaluated the safety and efficacy of venetoclax and obinutuzumab (G) in comparison with chlorambucil (C) and obinutuzumab in patients with IL in an open-label, multicenter, run-in phase of a randomized trial. Venetoclax was administered orally in a ramp-up dose fashion (20, 50, 100, 200, up to 400 $\mathrm{mg}$ ) starting on the 22nd day of the first cycle. Obinutuzumab (G) was given IV starting with 100, 900, 1,000, and $1,000 \mathrm{mg}$ on days one, two, eight, and 15 , respectively, followed by $1,000 \mathrm{mg}$ on day one for cycles two to six. Initially, six cycles of venetoclax plus obinutuzumab were given, followed by six cycles of venetoclax monotherapy. The most common adverse events observed were infusion-related reactions (75\%), neutropenia (67\%), infections (67\%), pruritus (58\%), and diarrhea (50\%). Grade 3-4 adverse events included neutropenia (58\%), infections (17\%), and thrombocytopenia (17\%). Laboratory TLS was seen in $17 \%$ of the patients with no evidence of clinical TLS. The ORR was 100\%, with CR in $58 \%$ of the patients and progression-free survival (PFS) in 100\%, evaluated at the end of 15 months, indicating that the method was effective and well-tolerated; and this trial has continued [48].

Flinn et al. conducted another phase $1 \mathrm{~b}$ trial (2015) evaluating the efficacy of venetoclax and obinutuzumab combination therapy in R/R or 1L patients with CLL. Treatment was started with either schedule A (VEN) or schedule $B(G)$ with a three+three design and 100-600 mg/day of venetoclax cohorts, given in gradual rampup fashion. Combination therapy continued for six cycles, followed by venetoclax monotherapy in R/R patients until disease progression and an additional six months of venetoclax monotherapy in 1L patients. Infectious adverse events and diarrhea were seen in 50\% of patients, while infusion-related reactions and neutropenia was seen in $40 \%$ and $37 \%$ of the patients, respectively. Grade 3 and grade 4 neutropenia was seen in $34 \%$, and $12 \%$ of the patients, respectively, and laboratory TLS was observed in $12 \%$ with no clinical TLS seen. One death in cohort 1, with R/R CLL, occurred secondary to acute respiratory failure. The ORR, evaluated in 17 patients, was found to be $100 \%$ and $\mathrm{CR} /$ complete remission with incomplete hematologic response (CRi) was seen in $23.5 \%$ of the patients. This again confirmed the safety and tolerability of VEN $+\mathrm{G}$ in both R/R and $1 \mathrm{~L}$ patients [49].

A phase Ib clinical trial was undertaken on 49 patients with relapsed or refractory CLL or SLL. Venetoclax was used in combination with rituximab. Venetoclax was started with a once-daily dose of $20 \mathrm{or} 50 \mathrm{mg}$, increased in a ramp-up fashion to final-cohort doses of 200-600 mg/day in five dose-escalation cohorts $(\mathrm{n}=41)$, while $400 \mathrm{mg} /$ day in safety-expansion cohort $(\mathrm{n}=8)$, tailed by rituximab, for a total of six doses given every four weeks. Of note, $92 \%$ and $59 \%$ of patients had received prior rituximab and fludarabine therapies, respectively. Patients having rituximab-refractory (R-ref) and fludarabine-refractory (F-ref) disease were $29 \%$ and $18 \%$, respectively. Twelve patients refused to continue the study: six because of PD (five were Richter's transformation), three due to adverse events [neuropathy, TLS, and myelodysplasia (heavily pretreated and hypocellular marrow at study entry; patient achieved minimal residual disease (MRD)negative complete remission (CR) with incomplete marrow recovery (CRi) and proceeded to transplant)]. Three withdrew consent (one after achieving MRD-negative CR). The most common adverse events seen in more than $25 \%$ of treatment subjects were neutropenia (55\%), diarrhea (53\%), nausea (49\%), and upper respiratory infection (45\%). Grade 3-4 adverse events seen in more than $10 \%$ of treatment subjects were neutropenia (53\%), thrombocytopenia (16\%), anemia (14\%), febrile neutropenia (12\%), and leukopenia (10\%). One death occurred due to TLS, after which the protocol was modified. ORR was $86 \%$ in all treatment subjects, $89 \%$ in del (17p), 56\% in F-ref, 84\% in IGHV-unmutated. The PFS measured at 12 months' duration for all patients, del (17p), F-ref, and IGHV-unmutated was 87\%, 89\%, 56\%, and 83\%, respectively, while PFS at 24 months was $84 \%, 78 \%, 56 \%$, and $83 \%$, respectively. The overall survival rate at 12 months was $94 \%$, $89 \%, 89 \%$, and $89 \%$, respectively, indicating that this combination therapy is active and highly responsive with a tolerable safety profile (Table 2) [50].

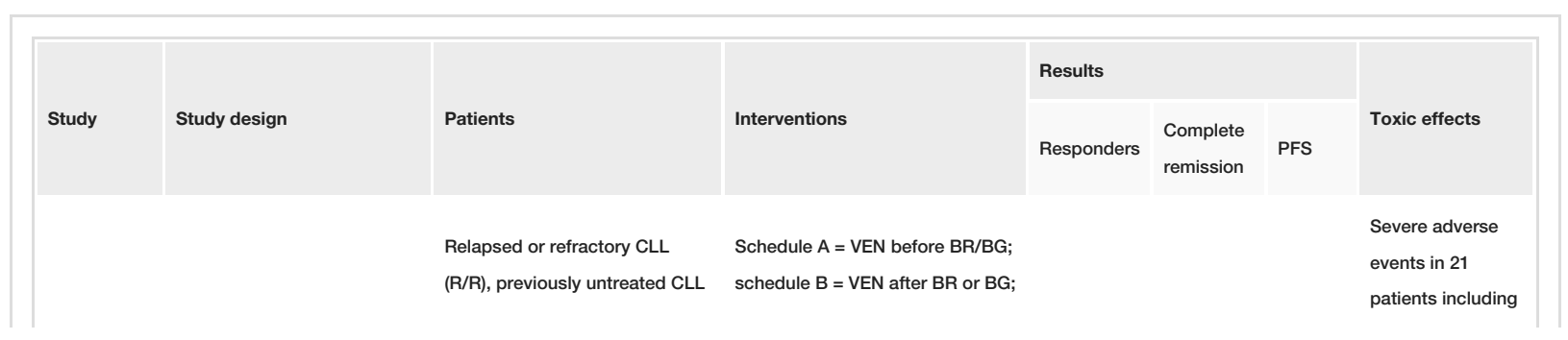




\section{Cureus}

\begin{tabular}{|c|c|c|c|c|c|c|c|}
\hline $\begin{array}{l}\text { Stilgenbauer } \\
\text { et al. (2016) } \\
{[47]}\end{array}$ & $\begin{array}{l}\text { Venetoclax with } \\
\text { bendamustine/rituximab } \\
\text { (VEN+BR) or } \\
\text { bendamustine/obinutuzumab } \\
\text { (VEN+BG); phase lb } \\
\text { (recruiting) }\end{array}$ & $\begin{array}{l}\text { (1L); VEN+BR (R/R, } n=30) \text {, } \\
\text { evaluated } n=24 \text {, del 17p }(25 \%) \text {, } \\
\text { del 11q }(29 \%) \text {, del 13q }(54 \%) ; \\
\text { VEN+BR (1L, } n=17) \text {, evaluated } \\
n=15 \text {, Del 17p (13\%), del 13q } \\
\text { (67\%); VEN+BG (1L, } n=8) \text {, } \\
\text { evaluated } n=5 \text {, del 17p (20\%), } \\
\text { del 11q }(20 \%) \text {, del 13q ( } 80 \%)\end{array}$ & $\begin{array}{l}47 \text { patients received VEN+BR: } 30 \\
\text { R/R (12 on schedule A at doses } \\
100-400 \mathrm{mg}, 18 \text { on schedule b, all } \\
\text { at } 400 \mathrm{mg} \text { ), and } 171 \mathrm{~L} \text { (all at } 400 \\
\mathrm{mg}: \text { six on schedule A, } 11 \text { on } \\
\text { schedule B). Eight patients } \\
\text { received VEN+BG: all on } \\
\text { schedule B at } 400 \mathrm{mg}\end{array}$ & $\begin{array}{l}\text { VEN+BR } \\
\text { (R/R) } 96 \%, \\
\text { VEN+BR } \\
\text { (1L) } 100 \%, \\
\text { VEN+BG } \\
\text { (1L) } 100 \%\end{array}$ & $\begin{array}{l}\text { VEN+BR } \\
\text { (R/R) } 20 \%, \\
\text { VEN+BR } \\
\text { (1L) } 43 \%, \\
\text { VEN+BG } \\
\text { (1L) } 43 \%\end{array}$ & & $\begin{array}{l}\text { erythema, febrile } \\
\text { neutropenia, } \\
\text { vomiting, nausea, } \\
\text { and infection } \\
(43 \% \mathrm{R} / \mathrm{R} \\
\text { VEN+BR, 35\% 1L } \\
\text { VEN+BR, 25\% 1L } \\
\text { VEN+BG) }\end{array}$ \\
\hline $\begin{array}{l}\text { Fischer et al. } \\
\text { (2016) [48] }\end{array}$ & $\begin{array}{l}\text { Venetoclax plus } \\
\text { obinutuzumab (VEN+G); } \\
\text { open-label, multicenter, run- } \\
\text { in phase of phase III } \\
\text { randomized trial }\end{array}$ & $\begin{array}{l}\text { Previously untreated CLL (1L) } \\
(n=12), \text { cytogenetic }(n=8) \text {, del } \\
17 p(25 \%) \text {, del 11q (25\%), TP53- } \\
\text { mutated }(25 \%) \text {, TP53 deleted } \\
(25 \%), \text { IGHV-mutated (evaluated } \\
n=7),(14 \%)\end{array}$ & $\begin{array}{l}\text { Six cycles (VEN+G) followed by } \\
\text { six cycles (VEN) I/V G ( } 100,900 \text {, } \\
1,000 \mathrm{mg} \text { on day one, two, eight, } \\
\text { and } 15 \text { of cycle one; on day one, } \\
1,000 \mathrm{mg} \text { for two to six cycles) } \\
\text { VEN oral in ramp-up dose weekly } \\
\text { starting at day } 22 \text { of cycle one } \\
(20,50,100,200, \text { and } 400 \mathrm{mg})\end{array}$ & $100 \%$ & $58 \%$ & $\begin{array}{l}100 \% \text { at } \\
15 \\
\text { months }\end{array}$ & $\begin{array}{l}\text { Neutropenia } \\
(67 \%) \text {, infections } \\
(67 \%) \text {, diarrhea } \\
(50 \%) \text {, infusion- } \\
\text { related reaction } \\
(75 \%) \text {, pruritus } \\
(58 \%) \text {. Grade } 3-4 \\
\text { adverse events } \\
\text { included } \\
\text { neutropenia } \\
(58 \%) \text {, infections } \\
(17 \%) \text {, and } \\
\text { thrombocytopenia } \\
(17 \%)\end{array}$ \\
\hline $\begin{array}{l}\text { Flinn et al. } \\
\text { (2015) [49] }\end{array}$ & $\begin{array}{l}\text { Venetoclax plus } \\
\text { obinutuzumab }(\mathrm{VEN}+\mathrm{G}) \text {, } \\
\text { phase lb, phase } 3 \text { continued }\end{array}$ & $\begin{array}{l}N=32 \text {, relapsed or refractory } \\
C L L(R / R) n=26 \text {, previously } \\
\text { untreated } C L L(1 L) n=6\end{array}$ & $\begin{array}{l}\text { Three+three design with } 100-600 \\
\mathrm{mg} / \text { day venetoclax cohorts }\end{array}$ & $\begin{array}{l}100 \% \\
\text { among } 17 \\
\text { evaluated } \\
\text { patients }\end{array}$ & $\begin{array}{l}23.5 \% \\
\text { among } 17 \\
\text { evaluated } \\
\text { patients }\end{array}$ & - & $\begin{array}{l}\text { Any infectious } \\
\text { adverse event } \\
(50 \%) \text {, diarrhea } \\
(50 \%) \text {, infusion- } \\
\text { related reactions } \\
(40 \%) \text {, } \\
\text { neutropenia } \\
(37 \%) . \text { Grade } 3 \\
\text { neutropenia } \\
(34 \%) \text {, grade } 4 \\
\text { neutropenia } \\
(12 \%)\end{array}$ \\
\hline $\begin{array}{l}\text { Ma et al. } \\
\text { (2015) [50] }\end{array}$ & $\begin{array}{l}\text { Venetoclax plus rituximab } \\
\text { (VEN+R), phase lb }\end{array}$ & $\begin{array}{l}\mathrm{N}=49 \text {, relapsed or refractory } \\
\mathrm{CLL}(48) \text { or SLL (1), R-ref } 29 \% \text {, } \\
\text { F-ref } 18 \% \text {, del(17p) } 18 \% \text {, IGHV- } \\
\text { unmutated } 39 \%\end{array}$ & $\begin{array}{l}\text { VEN (per day } 20 / 50 \mathrm{mg} \text { ) to final } \\
\text { cohort doses }(200-600 \mathrm{mg} \text { per } \\
\text { day) tailed by } \mathrm{R} \text {, total six doses in } \\
\text { every four weeks, final } 400 \mathrm{mg} \\
\text { selected to move forward }\end{array}$ & $\begin{array}{l}\text { ORR } 86 \%, \\
\text { BM MRD- } \\
\text { negative } \\
53 \%, \text { F-ref } \\
56 \%, \\
\text { deletion } \\
17 p \text { CLL } \\
\text { (89\%), } \\
\text { IGHV- } \\
\text { unmutated } \\
\text { (84\%) }\end{array}$ & $\begin{array}{l}\text { CR 41\%, } \\
\text { BM MRD- } \\
\text { negative } \\
75 \%, \text { F-ref } \\
44 \%, \\
\text { deletion } \\
17 p \text { CLL } \\
(33 \%), \\
\text { IGHV- } \\
\text { unmutated } \\
(37 \%)\end{array}$ & $\begin{array}{l}\text { PFS } \\
87 \% \text { at } \\
12 \\
\text { months, } \\
\text { PFS } \\
84 \% \text { at } \\
24 \\
\text { months, } \\
\text { overall } \\
\text { survival } \\
\text { (OS) } \\
94 \% \text { at } \\
12 \\
\text { months }\end{array}$ & $\begin{array}{l}\text { One fatal TLS } \\
\text { prior to protocol } \\
\text { modification, } \\
\text { grade } 3 / 4 \text { adverse } \\
\text { events in }>10 \% \text { of } \\
\text { pts: neutropenia } \\
(53 \%) \text {, } \\
\text { thrombocytopenia } \\
(16 \%) \text {, anemia } \\
(14 \%) \text {, and febrile } \\
\text { neutropenia } \\
(12 \%)\end{array}$ \\
\hline
\end{tabular}

\section{TABLE 2: Responders in venetoclax combination therapy in CLL patients}

CLL: chronic lymphocytic leukemia; SLL: small lymphocytic lymphoma; IGHV: immunoglobulin heavy-chain variable region; TP53: tumor protein p53; ORR: overall response rate; TLS: tumor lysis syndrome; VEN: venetoclax; B: bendamustine; R: rituximab; G: obinutuzumab; PFS: progressionfree survival; R-ref: rituximab-refractory; F-ref: fludarabine-refractory; MRD: minimal residual disease

\section{Conclusions}

Increased awareness and knowledge of CLL biology has enabled the synthesis of novel therapies that target specific steps of molecular pathways that facilitate tumor cell survival. Venetoclax is the first BH3 mimetic drug and a selective BCL-2 inhibitor that has received FDA approval. The main advantage of venetoclax over other agents is that it has a high binding affinity for BCL-2 receptors and it very selectively inhibits BCL-2, maintaining anti-apoptotic activity in cancer cells. Moreover, monotherapy with this agent facilitates a rapid reduction in the disease burden with an overall good response rate. This drug has proved to provide 
good therapeutic responses in CLL patients irrespective of the presence of adverse clinical or genetic features, including in patients with relapsed or refractory forms of CLL. We believe that the emergence of novel combination therapies, including venetoclax and immunotherapy, will transform the treatment landscape for patients with relapsed CLL, particularly those with (del 17p) CLL, which carries a very poor prognosis.

\section{Additional Information \\ Disclosures}

Conflicts of interest: In compliance with the ICMJE uniform disclosure form, all authors declare the following: Payment/services info: All authors have declared that no financial support was received from any organization for the submitted work. Financial relationships: All authors have declared that they have no financial relationships at present or within the previous three years with any organizations that might have an interest in the submitted work. Other relationships: All authors have declared that there are no other relationships or activities that could appear to have influenced the submitted work.

\section{References}

1. Elter T, Hallek M, Engert A: Fludarabine in chronic lymphocytic leukemia . Expert Opin Pharmacother. 2006, 7:1641-51. 10.1517/14656566.7.12.1641

2. DeSantis CE, Lin CC, Mariotto AB, et al.: Cancer treatment and survivorship statistics, 2014 . CA Cancer J Clin. 2014, 64:252-71. 10.3322/caac.21235

3. Siegel RL, Miller KD, Jemal A: Cancer statistics, 2015. CA Cancer J Clin. 2015, 65:5-29. 10.3322/caac.21254

4. Redaelli A, Laskin BL, Stephens JM, Botteman MF, Pashos CL: The clinical and epidemiological burden of chronic lymphocytic leukaemia. Eur J Cancer Care (Engl). 2004, 13:279-87. 10.1111/j.13652354.2004.00489.x

5. Hallek M, Cheson BD, Catovsky D, et al.: Guidelines for the diagnosis and treatment of chronic lymphocytic leukemia: a report from the International Workshop on Chronic Lymphocytic Leukemia updating the National Cancer Institute-Working Group 1996 guidelines. Blood. 2008, 111:5446-56. 10.1182/blood-200706-093906

6. Yamamoto JF, Goodman MT: Patterns of leukemia incidence in the United States by subtype and demographic characteristics, 1997-2002. Cancer Causes Control. 2008, 19:379-90. 10.1007/s10552-0079097-2

7. Eichhorst BF, Busch R, Stilgenbauer S, et al.: First-line therapy with fludarabine compared with chlorambucil does not result in a major benefit for elderly patients with advanced chronic lymphocytic leukemia. Blood. 2009, 114:3382-91. 10.1182/blood-2009-02-206185

8. Eichhorst B, Robak T, Montserrat E, Ghia P, Hillmen P, Hallek M, Buske C: Chronic lymphocytic leukaemia: ESMO Clinical Practice Guidelines for diagnosis, treatment and follow-up. Ann Oncol. 2015, 26:78-84. 10.1093/annonc/mdv303

9. Rivlin N, Brosh R, Oren M, Rotter V: Mutations in the p53 tumor suppressor gene. Genes Cancer. 2011, 2:466-74. 10.1177/1947601911408889

10. Huang Y, Wu JZ, Li JY, Xu W: Know the enemy as well as the weapons in hand: the aberrant death pathways and therapeutic agents in chronic lymphocytic leukemia. Am J Cancer Res. 2015, 5:2361-75.

11. Papakonstantinou G, Verbeke C, Hastka J, Bohrer M, Hehlmann R: Bcl-2 expression in non-Hodgkin's lymphomas is not associated with bcl-2 gene rearrangements. Br J Haematol. 2001, 113:383-90. 10.1046/i.1365-2141.2001.02727.x

12. Ott CJ, Federation AJ, Kasar S, Klitgaard JL, Fernandes SM, Brown JR, Bradner JE: Enhancer landscapes reveal transcription factor network dependencies in chronic lymphocytic leukemia. Blood. 2015, 126:436. 10.1182/blood.V126.23.436.436

13. Awan FT, Kay NE, Davis ME, et al.: Mcl-1 expression predicts progression-free survival in chronic lymphocytic leukemia patients treated with pentostatin, cyclophosphamide, and rituximab. Blood. 2009, 113:535-7. 10.1182/blood-2008-08-173450

14. Pekarsky Y, Zanesi N, Croce CM: Molecular basis of CLL. Semin Cancer Biol. 2010, 20:370-6. 10.1016/j.semcancer.2010.09.003

15. Doménech E, Gómez-López G, Gzlez-Peña D, et al.: New mutations in chronic lymphocytic leukemia identified by target enrichment and deep sequencing. PLoS One. 2012, $7:$ :e38158. 10.1371/journal.pone.0038158

16. Adams JM, Cory S: The Bcl-2 apoptotic switch in cancer development and therapy . Oncogene. 2007, 26:1324-37. 10.1038/sj.onc.1210220

17. Youle RJ, Strasser A: The BCL-2 protein family: opposing activities that mediate cell death . Nat Rev Mol Cell Biol. 2008, 9:47-59. 10.1038/nrm2308

18. Roberts AW, Davids MS, Pagel JM, et al.: Targeting BCL2 with venetoclax in relapsed chronic lymphocytic leukemia. N Engl J Med. 2016, 374:311-22. 10.1056/NEJMoa1513257

19. Roberts AW, Seymour JF, Brown JR, et al.: Substantial susceptibility of chronic lymphocytic leukemia to BCL2 inhibition: results of a phase I study of navitoclax in patients with relapsed or refractory disease. J Clin Oncol. 2012, 30:488-96. 10.1200/JCO.2011.34.7898

20. Stilgenbauer S, Eichhorst B, Schetelig J, et al.: Venetoclax in relapsed or refractory chronic lymphocytic leukaemia with 17p deletion: a multicentre, open-label, phase 2 study. Lancet Oncol. 2016, 17:768-78. 10.1016/S1470-2045(16)30019-5

21. Wilson WH, O'Connor OA, Czuczman MS, et al.: Navitoclax, a targeted high-affinity inhibitor of BCL-2, in lymphoid malignancies: a phase 1 dose-escalation study of safety, pharmacokinetics, pharmacodynamics, and antitumour activity. Lancet Oncol. 2010, 11:1149-59. 10.1016/S1470-2045(10)70261-8 
22. Souers AJ, Leverson JD, Boghaert ER, et al.: ABT-199, a potent and selective BCL-2 inhibitor, achieves antitumor activity while sparing platelets. Nat Med. 2013, 19:202-8. 10.1038/nm.3048

23. Salem AH, Agarwal SK, Dunbar M, Enschede SL, Humerickhouse RA, Wong SL: Pharmacokinetics of venetoclax, a novel BCL-2 inhibitor, in patients with relapsed or refractory chronic lymphocytic leukemia or non-Hodgkin's lymphoma. J Clin Pharmacol. 2017, 57:484-92. 10.1002/jcph.821

24. FDA: Venclexta (venetoclax tablets) prescribing information . (2016). Accessed: July 20, 2018: http://www.accessdata.fda.gov/drugsatfda_docs/label/2016/208573s000lbl.pdf.

25. Jones AK, Freise KJ, Agarwal SK, Humerickhouse RA, Wong SL, Salem AH: Clinical predictors of venetoclax pharmacokinetics in chronic lymphocytic leukemia and non-Hodgkin's lymphoma patients: a pooled population pharmacokinetic analysis. AAPS J. 2016, 18:1192-202. 10.1208/s12248-016-9927-9

26. Ludden TM: Nonlinear pharmacokinetics: clinical implications . Clin Pharmacokinet. 1991, 20:429-46. 10.2165/00003088-199120060-00001

27. Salem AH, Agarwal SK, Dunbar M, Nuthalapati S, Chien D, Freise KJ, Wong SL: Effect of low- and high-fat meals on the pharmacokinetics of venetoclax, a selective first-in-class BCL-2 inhibitor. J Clin Pharmacol. 2016, 56:1355-61. 10.1002/jcph.741

28. Lee ID, Amidon GL: Pharmacokinetic Analysis: A Practical Approach . Technomic Publishing Company, Inc, Lancaster, PA; 1996.

29. Freise KJ, Jones AK, Eckert D, et al.: Impact of venetoclax exposure on clinical efficacy and safety in patients with relapsed or refractory chronic lymphocytic leukemia. Clin Pharmacokinet. 2017, 56:515-23. 10.1007/s40262-016-0453-9

30. Davids MS, Roberts AW, Seymour JF, et al.: Phase I first-in-human study of venetoclax in patients with relapsed or refractory non-Hodgkin lymphoma. J Clin Oncol. 2017, 35:826-33. 10.1200/JCO.2016.70.4320

31. Llambi F, Moldoveanu T, Tait SW, et al.: A unified model of mammalian BCL-2 protein family interactions at the mitochondria. Mol Cell. 2011, 44:517-31. 10.1016/j.molcel.2011.10.001

32. Czabotar PE, Lessene G, Strasser A, Adams JM: Control of apoptosis by the BCL-2 protein family: implications for physiology and therapy. Nat Rev Mol Cell Biol. 2014, 15:49-63. 10.1038/nrm3722

33. Cory S, Adams JM: The Bcl2 family: regulators of the cellular life-or-death switch . Nat Rev Cancer. 2002, 2:647-56. 10.1038/nrc883

34. Chen L, Willis SN, Wei A, et al.: Differential targeting of prosurvival Bcl-2 proteins by their BH3-only ligands allows complementary apoptotic function. Mol Cell. 2005, 17:393-403. 10.1016/j.molcel.2004.12.030

35. McDonnell TJ, Deane N, Platt FM, Nunez G, Jaeger U, McKearn JP, Korsmeyer SJ: bcl-2-immunoglobulin transgenic mice demonstrate extended B cell survival and follicular lymphoproliferation. Cell. 1989, 57:7988. 10.1016/0092-8674(89)90174-8

36. Strasser A, Harris AW, Bath ML, Cory S: Novel primitive lymphoid tumours induced in transgenic mice by cooperation between myc and bcl-2. Nature. 1990, 348:331-3. 10.1038/348331a0

37. Schmitt CA, Lowe SW: Bcl-2 mediates chemoresistance in matched pairs of primary $\mathrm{E}(\mathrm{mu})$-myc lymphomas in vivo. Blood Cells Mol Dis. 2001, 27:206-16. 10.1006/bcmd.2000.0372

38. Oltersdorf T, Elmore SW, Shoemaker AR, et al.: An inhibitor of Bcl-2 family proteins induces regression of solid tumours. Nature. 2005, 435:677-81. 10.1038/nature03579

39. Leverson JD: Chemical parsing: dissecting cell dependencies with a toolkit of selective BCL-2 family Inhibitors. Mol Cell Oncol. 2015, 3:e1050155. 10.1080/23723556.2015.1050155

40. Landau DA, Carter SL, Stojanov P, et al.: Evolution and impact of subclonal mutations in chronic lymphocytic leukemia. Cell. 2013, 152:714-26. 10.1016/j.cell.2013.01.019

41. Anderson MA, Deng J, Seymour JF, et al.: The BCL2 selective inhibitor venetoclax induces rapid onset apoptosis of CLL cells in patients via a TP53-independent mechanism. Blood. 2016, 127:3215-24. 10.1182/blood-2016-01-688796

42. Thijssen R, Geest CR, de Rooij MFM, et al.: Possible mechanisms of resistance to the novel BH3-mimetic ABT-199 in in vitro lymph node models of CLL - the role of Abl and Btk. Blood. 2013, 122:4188. 10.1182/blood.V122.21.4188.4188

43. Woyach JA, Johnson AJ: Targeted therapies in CLL: mechanisms of resistance and strategies for management. Blood. 2015, 126:471-7. 10.1182/blood-2015-03-585075

44. Oppermann S, Ylanko J, Shi Y, et al.: Identification of kinase inhibitors that overcome venetoclax resistance in activated CLL cells by high-content screening. Blood. 2016, 12:8-15. 10.1182/blood-2015-12-687814

45. Jones J, Mato A, Coutre S, et al.: Preliminary results of a phase 2, open-label study of venetoclax (ABT199/GDC-0199) monotherapy in patients with chronic lymphocytic leukemia relapsed after or refractory to ibrutinib or idelalisib therapy. Blood. 2015, 126:715. 10.1182/blood.V126.23.715.715

46. Fresquet V, Rieger M, Carolis C, García-Barchino MJ, Martinez-Climent JA: Acquired mutations in BCL2 family proteins conferring resistance to the BH3 mimetic ABT-199 in lymphoma. Blood. 2014, 123:4111-9. 10.1182/blood-2014-03-560284

47. Stilgenbauer S, Morschhauser F, Wendtner CM, et al.: Phase Ib study (GO28440) of venetoclax with bendamustine/rituximab or bendamustine/obinutuzumab in patients with relapsed/refractory or previously untreated chronic lymphocytic leukemia. Blood. 2016, 128:4393. 10.1182/blood.V128.22.4393.4393

48. Fischer K, Al-Sawaf O, Fink A, et al.: Safety and efficacy of venetoclax and obinutuzumab in patients with previously untreated chronic lymphocytic leukemia (CLL) and coexisting medical conditions: final results of the run-in phase of the randomized CLL14 trial (BO25323). Blood. 2016, 128:2054. 10.1182/blood.V128.22.2054.2054

49. Flinn IW, Brunvand M, Choi MY, et al.: Safety and efficacy of a combination of venetoclax (GDC-0199/ABT199) and obinutuzumab in patients with relapsed/refractory or previously untreated chronic lymphocytic leukemia - results from a phase 1b study (GP28331). Blood. 2015, 126:494. 10.1182/blood.V126.23.494.494

50. Ma S, Brander DM, Seymour JF, et al.: Deep and durable responses following venetoclax (ABT-199 / GDC0199) combined with rituximab in patients with relapsed/refractory chronic lymphocytic leukemia: results from a phase 1b study. Blood. 2015, 126:830. 10.1182/blood.V126.23.830.830 